\title{
Der Einsatz von Neuen Informations- und Kommunikations- Technologien zur Wissensvernetzung
}

\author{
Maximilian Senges
}

"We are drowning in information, but starving for knowledge"

John Naisbitt

\section{Abstract}

The following article highlights the main points of the diploma thesis "The utilization of New Information and Communication Technologies for Knowledge Networking”(,Der Einsatz von Neuen Informations- und Kommunikations-Technologien zur Wissensvernetzung“") which can be accessed at http://turku.wi-bw.tfh-wildau.de/ $\sim$ drmue/Diplomarbeiten/Senges_Max.pdf.

The Thesis researches and discusses the planning and launch of a community of practice at the United Nations Office for Project Services (UNOPS). The objective of this paper is to lay out a practical framework for "knowledge networking" by bringing together strategic, technological and procedural aspects and by illustrating the implementation of the framework with a empirical study at UNOPS. The work focuses on describing the right organisational and technological environment to share knowledge and expertise through New Information and Communication Technologies (NICT) in a global context. Further more it deals with computer mediated communication as a primary task of the modern knowledge worker and as prerequisite of effective "knowledge networking".

The case study at UNOPS describes the successful launch and practices of a international and intercultural knowledge network. Here the paper will not elaborate on technological aspects, because of the fast change of the performance and functionality, but will try to show the strategic and organisational implications. The case study displays the collective empowerment of practitioner groups to initialize and optimize their practices and working-tools. The results of this research indicate that the introduced collaborative network enables democratisation and transparency in corporate governance.

The paper suggests that being "networked" is a quality of successful organisations and that "knowledge networking" can play a substantive role in tackling the new challenges of globalized competition.

Der voliegende Beitrag stellt eine Zusammenfassung der Ergbnisse der Diplomarbeit „Der Einsatz von Neuen Informations- und Kommunikations-Technologien zur Wissensvernetzung“ dar. Die Arbeit kann unter http: //turku.wi-bw.tfh-wildau.de/ drmue/Diplomarbeiten/ Senges_Max.pdf eingesehen werden.

Die Arbeit untersucht und diskutiert die Planung und Einfuihrung einer Community of Practice in dem Büro für Projektservicedienstleistungen der Vereinten Nationen
(UNOPS). Das Ziel der Arbeit ist es, durch das Zusammenfuihren strategischer, technologischer und prozessorientierter Grundlagen einen anwendungsorientierten Gestaltungsrahmen für „Wissensvernetzung“vorzulegen, der anhand einer praxisorientierten Fallstudie illustriert wird. Der Fokus der Arbeit konzentriert sich besonders auf die Schaffung der Rahmenbedingungen, um Wissen und Expertise durch Neue Informations- und Kommunikations-Technologien (NIKT) in einem globalen Kontext verfuigbar zu machen. Außerdem beschäftigt sie sich mit den Prozessen computervermittelter Kommunikation als eine primäre Arbeitsaufgabe des modernen Wissensarbeiters und als Vorraussetzung einer effektiver „Wissensvernetzung“.

In der Fallstudie bei UNOPS wird die erfolgreiche Einfuihrung und Arbeitsweise eines internationalen, interkulturellen Wissensnetzes beschrieben. Dabei geht es nicht darum, die technologischen Grundlagen der NIKTWerkzeuge zu erläutern, da der strategisch orientierte Horizont nicht mit den kurzen Entwicklungsschritten der technologischen Werkzeuge vermengt werden soll. Die Fallstudie soll die Möglichkeit der kollektiven Einflussnahme der vernetzten Mitarbeiter eines Fachbereichs, auf ihre Arbeitsweisen und Arbeitsmittel darstellen. Die Ergebnisse der Arbeit legen nahe, dass diese neuen kollaborativen Handlungsfelder der Mitarbeiter zu einer Demokratisierung und Transparenz innerhalb der Organisation führen.

In der Arbeit soll gezeigt werden, dass die interne Vernetzung zu einer wichtigen Qualitätsbeschreibung erfolgreicher Organisationen wird und dass „Wissensvernetzung“ einen entscheidenden Beitrag zur Bewältigung neuer Wettbewerbsanforderungen leistet.

\section{Einleitung}

Menschen arbeiten in Gruppen und schaffen durch kollegiale Zusammenarbeit eine Gemeinschaft, in der sich Talente ergänzen und Schwächen ausgleichen lassen. Durch die Globalisierung wurden diese Arbeitsgemeinschaften räumlich verteilt und der einzelne Mitarbeiter von seinen Kollegen isoliert. Die Arbeit beschreibt einen Ansatz, bei dem Neue Informations- und Kommunikations-Technologien (NIKT) eingesetzt werden, um moderne Arbeitsgemeinschaften in Form von kollaborativen „Wissensnetzen“ zu schaffen. Diese ermöglichen den Mitarbeiter Probleme als Team zu lösen.

Die Arbeit beschäftigt sich praxisorientiert mit dem Thema organisationsinterner Vernetzung von Wissen und bezieht sich dabei auf Wissensmanagement, organisatorische Informationsverbreitung und -bereitstellung 
sowie computerunterstuitzte Gruppenarbeit. Es geht um die Einfuihrung so genannter Communities of Practice (CoP), die Mitarbeiter des gleichen Arbeitsbereichs mit Hilfe von Neuen Informations- und KommunikationsTechnologien (NIKT) zusammenbringen. In den CoP übernehmen die Wissensarbeiter als Gruppe Verantwortung für Aktivitäten und Entwicklungen in ihrem Arbeitsbereich, wobei ein besonderer Stellenwert der Aufgabe zukommt, gemeinsames Wissen zu vernetzen. Aber was genau ist Wissensvernetzung?

\section{Wissensvernetzung}

Der Begriff Wissensvernetzung ist bisher weder in Forschung noch in der Praxis besonders etabliert. Wissensnetze waren aber z. B. das zentrale Thema des am Wissenschaftszentrum Nordrhein-Westfalen abgehaltenen Workshops „Strategische Wissensnetze: Wie Unternehmen die Ressource Wissen nutzen“ [1]. Er wurde gewählt, da er die Interdependenz der Teilnehmer verdeutlicht und durch den aktiven, prozesshaften Charakter der Vernetzung die Notwendigkeit der Interaktivität der Teilnehmer beschreibt.

Menschen arbeiten und leben seit jeher vernetzt und tauschen Wissen aus. Zwei Aspekte verschaffen der Wissensvernetzung jedoch eine neue Bedeutung:

Erstens, durch die wachsende Bedeutung der Ressource Wissen bzw. ihrer gespeicherten externalisierten Form Information steigt der Bedarf an bewusst gesteuerten Abläufen. Das in jeder Organisation exponentielle Wachstum der digital gespeicherten Datenmenge ist jedoch nicht gleichbedeutend mit höherem Informationsbestand oder gar Wissen. Die NIKT bieten zwar eine große Chancen für die Entwicklung zu einer besser informierten und mehr wissenden Organisation, doch lässt sich diese nicht auf die technische Beschaffenheit der NIKT reduzieren, sondern stellt vor allem neue Anforderungen an die organisatorischen Rahmenbedingungen und die Arbeitsweise des einzelnen Mitarbeiters. Andreas Boes schreibt dazu in der Frankfurter Rundschau vom 21. März 1997 (S. 22, Spalte 1): „Das Entstehen der Informationstechnik muss als materialisierter Ausdruck einer historischen Entwicklung der , Informatisierung der Arbeit' gesehen werden, deren Beginn viel weiter als der Einsatz der ersten Computer zurückliegt. Menschliche Arbeitsprozesse sind dieser Überlieferung zufolge niemals nur stoffliche Tätigkeiten, sie sind immer zugleich auch wissensverarbeitende Prozesse“, die im Laufe der Geschichte immer komplexer geworden sind. Der momentan von den Industrienationen vollzogene Übergang zur so genannten Informationsgesellschaft beinhaltet nach Boes „die Entstehung einer qualitativ neuartigen Informationsebene der Produktionsprozesse, und wird [seiner Meinung nach] einen tiefgreifenden Umbruch der Arbeit mit sich bringen."

Zweitens ermöglichen NIKT idealerweise den Zugriff auf das den gesamten Informationsbestand der Organisation umfassende Enterprise Content Management (ECM)-System von jedem Arbeitsplatz. Der digitale InformationsRaum, der durch NIKT und speziell durch ECM-Systeme entstanden ist, wird als Informations-Sphäre bezeichnet. Sie besteht aus - meist in Datenbanken gespeicherter - semantisch und logisch miteinander verbundener Information. Jeder Mitarbeiter kann sich aktiv an der Entwicklung dieser organisatorischen Wissensbasis, dem „Organisational Memory“, beteiligen und ist somit Teil der „Organisational Intelligence“, indem er Inhalte, Kommentare, Fragen und Antworten organisationsweit veröffentlicht [2].

\section{Neue Werkzeuge erfordern neue Arbeitsweise}

Neue Informations- und Kommunikations-Technologien (NIKT) werden als Werkzeuge eingesetzt, um innerhalb der informationsorganisatorischen Strukturen und der organisationskulturellen Rahmenbedingungen die produktive und effektive (Zusammen-)Arbeit zwischen Wissensarbeitern zu ermöglichen. In der Diplomarbeit stehen der Sinn und der Einsatz der Werkzeuge, nicht aber deren Entwicklung im Mittelpunkt der Betrachtungen.

Die Aufgabe speziell der Informations-Manager (aber auch der Wissensmanager) ist es, eine hohe Qualität, eine hohe Verfügbarkeit und Zugänglichkeit der Informationen zu sichern. Diese Aufgabe wird heutzutage vom Informations- und Kommunikations-Management beforscht und durch Bereitstellung einer Informationsinfrastruktur und einer Informationsarchitektur angegangen.

Infolge dieser Demokratisierung im Bezug auf Informationszugriff und Informationsverbreitung, also des Zugangs zu Medien, Inhalten, Ressourcen und Funktionen, ist Wissensvernetzung eine Aufgabe für jeden Mitarbeiter. Informationen und Wissen effizient auszutauschen erfordert neue Fähigkeiten im Bezug auf die Nutzung der NIKT die im Kapitel 4 der Diplomarbeit unter „Computer unterstuitzte Gruppenarbeit“ dargestellt werden. Dieser Artikel wird in den nächsten Absätzen die Veränderungen der Arbeitsumwelt und der Organisationsform beschreiben.

\section{Höhere Interdependenz der Mitarbeiter}

Die durch die Industrielle Revolution geschaffene technologische Entwicklung speziell in den Bereichen Informations- und Kommunikations-Technologien und Logistik haben durch extrem gesunkene Transaktions-, Kommunikations- und Transportkosten die Wirtschaftsräume der Welt zusammenwachsen lassen. Dies fuihrt erstens dazu, dass Organisationen ihren Aufbau und ihre Arbeitsweise den veränderten Verhältnissen anpassen müssen. Dies und die Entwicklung einer immer größeren und komplexeren Organisation hat neue Organisationsformen nötig gemacht, die im folgenden Abschnitt beschrieben werden. Diese moderne Organisationsformen schaffen eine Interdependen $\mathrm{z}$ (Vernetzung) der Aktivitäten der über den ganzen Globus verteilten Mitarbeiter, die jedoch nur durch effektive Steuerung der Kommunikation und Kooperation ausreichend realisiert werden kann. 


\section{Verschiebungen in der Organisations- struktur}

Wissen ist neben den Produktionsfaktoren Boden, Kapital und Arbeitskraft zu einer kritischen Ressource geworden, deren Aufstieg oft unbemerkt zu enormen Verschiebungen in der Organisationsstruktur fuihrt. Da die Neuen Informations- und Kommunikations-Technologien die traditionellen, starren Berichtswege umgehen und den Austausch zwischen den Mitarbeitern fördern, entsteht eine Kommunikations-Matrix, die bewusst eingesetzt zu höherer Transparenz und Koordination bei Entscheidungen führt. Der erhöhte Bedarf an Kommunikation und Koordinierung fuihrte unter anderem zu dem Trend hin zu einer Matrix Organisationsform, bei der neben den vertikalen funktionalen Einheiten horizontale thematische bzw. projektbezogene Bindungen entstehen.

Eine Matrixorganisation kann durch die Ausbildung mehrerer Partialzentren ein integriertes Netzwerk mit verteilten, aber interdependenten und spezialisierten Kernkompetenzen zur Überwindung des Dilemmas von zentraler versus dezentraler Steuerung bzw. Integration versus Differenzierung beitragen (Perlitz 2000). Durch den Einsatz von NIKT können die Partialzentren ihre Aktivitäten auch direkt untereinander koordinieren und Aufgaben jeweils an die Einheit verlagern, die die besten Kompetenzen dafür besitzt. Die Stärke dieser Struktur beruht nach Perlitz insbesondere auf folgenden Charakteristika:

- differenzierte Beiträge der dezentralen Einheiten zu integrierten und weltweiten Aktivitäten,

- weltweit verstreute, interdependente und spezialisierte Werte und Fähigkeiten sowie

- gemeinsam entwickeltes Wissen zur weltweiten Nutzung durch alle Unternehmenseinheiten.

Durch das Zusammenspiel von Muttergesellschaft und dezentralisierten Büros entsteht eine polyzentrische Struktur, in der Netzwerkmitglieder in Abhängigkeit von den Kriterien „strategische Bedeutung der lokalen Einheiten“ und „Niveau der lokalen Ressourcen und Kompetenzen“ unterschiedliche Rollen einnehmen können. Die wichtigste Funktion haben die Einheiten in der Rolle des „strategischen Führers“. Sie übernehmen für ein bestimmtes Teilgebiet die strategische Gesamtverantwortung und koordinieren die Aktivitäten der anderen Einheiten [3]. Diese Form der polyzentrischen Struktur als Ausprägung der Matrixorganisation bildet allerdings viele Machzentren aus, die um einen Führungsanspruch in bestimmten Bereichen wetteifern. Daher muss die Machtverteilung immer wieder neu verhandelt werden. Macht und Verhandlung besitzen im Bereich der Politologie eine lange Forschungstradition, deren Ergebnisse im Bereich Führung sich auch auf andere Organisationen übertragen lassen.

Nach diesen wissenschaftlich theoretischen Ausfuihrungen seien im Folgenden der Ablauf und die Ergebnisse der Fallstudie beschrieben.

\section{Fallstudie bei UNOPS}

Die besprochene Arbeit beschreibt die Einfuihrung einer Community of Practice im Arbeitsbereich Beschaffung des United Nations Office for Project Services (UNOPS) und betrachtet dabei vor allem die produktive Nutzung der NIKT für den Austausch und die Zusammenarbeit räumlich getrennter Gruppenmitglieder.

Durch die Verteilung von Spezialisten auf Büros in der ganzen Welt und die damit einhergehende räumliche und zeitliche Trennung der Mitarbeiter sind der Informationsaustausch und die Koordination zwischen den Beschaffungsexperten nicht ausreichend. Die mangelnde Abstimmung und das Nichtvorhandensein unternehmensweiter Arbeitsweisen (Practices) führen zu einer Intransparenz und Inkongruenz im Ablauf der einzelnen Beschaffungsprozesse für Mitarbeiter und Kunden. Diese Situation im Arbeitsfeld Beschaffung, das mit rund 500 Millionen Dollar Umsatz im Jahr ein wichtiges Standbein von UNOPS ist, sollte durch die Einfuihrung einer Community of Practice (CoP) verbessert werden.

Das Pilot-Projekt dauerte sechs Monate, wobei davon ca. vier Monate auf Analyse und Planung verwendet wurden. Dies ergibt eine Aktivität der Gruppe von zwei Monaten. Da innerhalb dieser zwei Monate bereits mehrere konkrete Resultate der Zusammenarbeit vorlagen und da alle Teilnehmer sich positiv über die Möglichkeiten, die die Community ihnen bot, äußerten, wurde beschlossen, die Community als dauerhafte Institution in die UNOPS-Arbeitswelt zu integrieren. Im Folgenden seien die wichtigesten Erfahrungen vorgestellt:

\section{Lessons Learned}

\subsection{WM-Aufklärungsarbeit \& Integration in Arbeits- umwelt}

Communities of Practice sind eine hervorragende Ergänzung der Organisationsstruktur und können bei zahlreichen konkreten Problemen einen Input bzw. Lösungsvorschläge der Betroffenen selbst hervorbringen. Da das Konzept bzw. die Arbeitsweise einer CoP fuir die meisten Mitarbeiter neu ist, muss speziell bei der Einfuihrung viel Aufklärungsarbeit geleistet werden.

Die Integration der CoP in die Arbeitsroutine der Mitarbeiter dauert mehrere Monate, während dieser Zeit ist der Einsatz eines Coaches bzw. eine hohe Aktivität des Moderators äußerst wichtig, um den Diskussionsfluss im Gang zu halten und zu qualitativ relevanten Ergebnissen zu kommen.

Wie in jeder Gruppe gab es auch in dieser CoP einige Teilnehmer, die sich und ihre (Macht-)Position durch die neue Form der verteilten und kollektiven Steuerung von Prozessen und Entscheidungen bedroht sahen. Der Umgang mit diesen destruktiven und extrem kritischen Teilnehmern ist sehr schwierig und erfordert Erfahrung und Standfestigkeit. Es hat sich bewährt, solche Themen im Projektteam zu besprechen und auf Kritik und Attacken mit einer argumentativ fundierten Nachricht des gesamten Teams zu antworten, die Beantwortung der Kritik durch andere Teilnehmer (oder u. U. Außen- 
stehende) anzuregen oder in extremen Fällen die Kritik einfach zu ignorieren. Macht und politisch motivierte Opposition zu Wissensnetzen sind eine von den Moderatoren explizit geäußerte Herausforderung bei der Einfuihrung der neuen Konzepte.

Als extrem wichtig wurde außerdem die Unterstuitzung des Direktors wahrgenommen, ohne die die Motivation der Teilnehmer, Zeit und Energie in den Prozess zu investieren, mit Sicherheit nicht so hoch gewesen wäre.

\subsection{Einfache NIKT und Projektcontrolling}

Sehr wichtig scheint, dass die Wissensvernetzung so eng wie irgend möglich mit den bestehenden Arbeitsweisen (Practice) verbunden wird. Die Zeit, die in die Wissensvernetzung investiert werden muss, ist für den Mitarbeiter meist schon schwer genug zu finden. Wenn dazu noch neue (womöglich komplexe) Abläufe erlernt werden müssen, strecken die meisten Mitarbeiter die Waffen. Dies gilt speziell bei der Wahl der NIKT-Werkzeuge.

Die Entwicklung der Intranet-Funktionalitäten gestaltete sich außerst schwierig, da der verantwortliche Programmierer in New York, auf Grund unkoordinierter Entwicklungsaufträge von mehreren Abteilungen, extrem belastet war. Auch nachdem die Projektleiter mit der ITAbteilung gesprochen hatte und die Mitarbeiter-Profile fuir den Programmierer Priorität hatten, wurde die Entwicklung nicht stringent bearbeitet. Es scheint daher fuir vergleichbare Aufgaben unvermeidbar, ein Projektcontrolling einzurichten.

\subsection{Besprechungen sind Produktivitätskiller}

Die Fallstudie hat gezeigt, dass die wenigsten Besprechungen nach den in Kapitel 3 und 4 beschriebenen Schemata vorbereitet, durchgefuihrt und nachbereitet werden. Es scheint enorm wichtig für die Wissensvernetzung und die Produktivitätssteigerung allgemein, offizielle Richtlinien und Standards bei der Durchfuihrung von Besprechungen (egal über welches Medium oder Face-to-face) einzufuihren.

Des Weiteren sollte versucht werden, so oft wie möglich soziale Kontakte (die die Vertrauensbasis heben) zwischen den Mitarbeitern zu ermöglichen. Hierzu sind Relax-Zonen, in denen sich die Wissensarbeiter in den nötigen Pausen treffen und kennen lernen können, sehr geeignet.

\section{Fazit}

Der Begriff Wissensmanagement taucht in letzter Zeit in immer mehr Unternehmensberichten auf und viele Unternehmensberater-Gutachten nennen Wissensmanagement als eine Schlüsselaktivität, um konkurrenzfähig zu bleiben. Meist bleibt es jedoch bei Lippenbekenntnissen, da unklar ist, wie die entsprechende Organisationskultur, organisatorisch-technologischen Rahmenbedingungen und die Arbeitsweise (siehe Abbildung 1) implementiert werden können, und da es sehr schwer ist, für Bemühungen um die Ressource Wissen ein Return on Investment nachzuweisen.

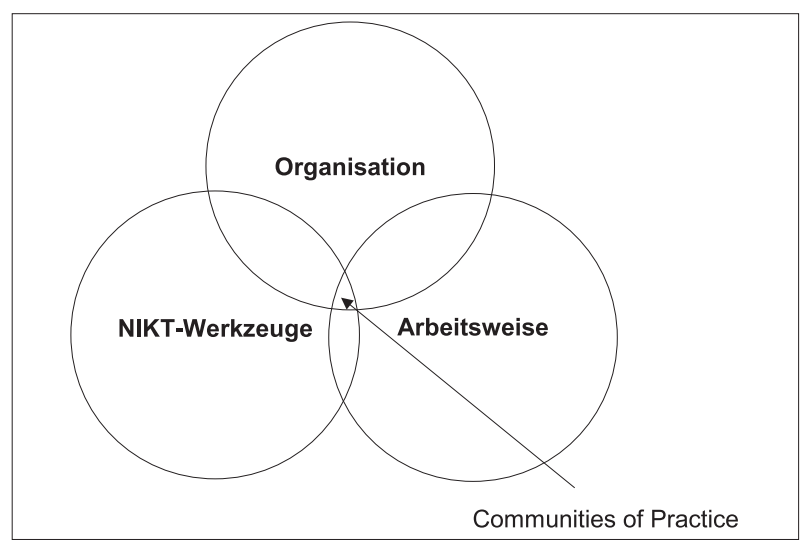

Abb. 1: Drei Komponenten der Wissensvernetzung

Die durch die Fallstudie gewonnene Erkenntnis ist, dass das Management von Wissen nur einen Teil der Herausforderung, Wissen in einer Organisation nutzbar zu machen, darstellt. Für den ganzheitlichen Ansatz „Wissen und Informationen besser zu verwerten" wurde in dieser Arbeit der Begriff Wissensvernetzung gewählt. Wissensvernetzung besteht aus drei Teilen, die zwar eng miteinander verbunden sind, aber doch eigenständige Erfordernisse haben.

\section{Organisation: der soziologisch-organisatorische Aspekt} Wissensnetze sind Gemeinschaften von Kollegen, die sich gegenseitig helfen und NIKT als Trägermedium zum Austausch von Ideen und Wissen in der NoosSphäre (siehe Einleitung) nutzen. Dieser Bereich versteht Wissensmanagement als einen sozialen und organisatorischen Prozess, der zwischen Menschen abläuft und nur innerhalb bestimmter organisatorischer Rahmenbedingungen und einer offenen und „familiären“ (vertrauensvollen) Organisationskultur funktionieren kann. Ziel des Wissensmanagements ist somit, den Mitarbeitern die Bedeutung von Wissensaustausch bewusst zu machen und Wissensnetze als Institution und Funktion in die Arbeitsweise der Organisation zu integrieren.

\section{NIKT-Werkzeuge: das Management und die Pflege der Prozesse und Strukturen der Informations-Sphäre} Die beiden Aufgaben, das Schaffen eines (1.) technologischen Informationssystems zum Kommunikation expliziten Wissens sowie das Bereitstellen der (2.) NIKT als Medium für impliziten Wissensaustausch (Sozialisation), schaffen die technologischen Rahmenbedingungen (Mittel). Diese Aufgaben werden im Informations- und Kommunikations-Management zusammengefasst. Kommunikations-Management beschäftigt sich mit der technologischen Infrastruktur sowie dem Anbieten von Kommunikationskanälen (Medien), die den zielgruppengenauen Austausch von Nachrichten ermöglichen. Das Informationsmanagement hat die Bereitstellung eines Systems, das die Informationen einer Organisation in einer Datenbank katalogisiert, archiviert, verwaltet und den Mitarbeitern zugänglich macht, zum Ziel. Bei beiden Aufgaben haben eine möglichst hohe Benutzerfreundlichkeit und eine Integration in den Arbeitsalltag der Wissensarbeiter Priorität. 


\section{Arbeitsweisen:}

Da NIKT in beiden Bereichen eine entscheidende Rolle spielen und von den Mitarbeiter neue Arbeitsweisen (Practice) und Arbeitsformen der Gruppenarbeit verlangen, ist es sehr sinnvoll, die Einführung eines Wissensvernetzungsprogramms durch Konzepte und Schulungsmaßnahmen im Bereich Computerunterstiitzte Gruppenarbeit zu untermauern. Dieser nur scheinbar banale Punkt wird in der Praxis besonders oft missachtet und fuihrt zum Scheitern. Dies sei im Folgenden weiter erläutert.

Sehr viele NIKT-Lösungen im Bereich Wissensmanagement scheitern an der Ablehnung der Menschen, die sie benutzen sollen. Dies liegt einerseits an schwierig zu bedienender Software und deren mangelhafter Qualität, und andererseits an der Tatsache, dass sehr viele Mitarbeiter keine ausreichenden Fähigkeiten im Einsatz der NIKT haben. Einzig E-Mail und das Internet werden in einer 1998 veröffentlichten Studie, die die Effektivität und Nuitzlichkeit von Software fuir das Wissensmanagement untersucht [4], als nuitzliches und integriertes Mittel zum Austausch von Wissen bewertet.

Wissensarbeiter haben meist eine sehr differenzierte Ausbildung in ihrem Fachbereich erhalten und beherrschen die Werkzeuge dieser Disziplin. In global agierenden und dezentralen Organisationen kommen aber, neben den arbeitsbereichspezifischen Werkzeugen, die NIKT-basierte Kooperation und Kommunikation hinzu, für die die wenigsten Wissensarbeiter eine spezielle Schulung erhalten haben. Bemuihungen wie der, während dem Pilotprojekt eingefuihrte, internationale ComputerFührerschein (International Computer Driving Licence - ICDL) schulen eben nicht die computerunterstuitzte Zusammenarbeit oder computervermittelte Kommunikation, sondern Grundlagen in MS Word und Excel. Dies zeigt ganz eindeutig, dass die meisten heutigen Wissensarbeiter nicht in der Lage sind, die relativ komplexen informationstechnologischen aber auch koordinatorischen Arbeitsabläufe beim gemeinsamen virtuellen Arbeiten zu meistern. Hinzu kommt, dass viele Mitarbeiter sich in einer global verteilten Community, die hauptsächlich virtuell arbeitet, nur schwer integrieren und „zu Hause“ fühlen können. Auch hierfür ist es sinnvoll, zumindest den Moderatoren und Projektleitern ein CSCW-Training mit Fokus auf virtueller Teambildung zu ermöglichen.

\section{Arbeitsteilung zwischen Wissensmanagement und IKM}

In der Praxis uiberschneiden sich die Aufgaben der Wissensmanager und der Informations- und Kommunikationsmanager in vielen Bereichen. Eine klar definierte Arbeits- und damit Verantwortungsverteilung ist aber elementar für die Kreation und Pflege qualitativ hochwertiger Wissensnetze. Die in der Diplomarbeit vertretene Arbeitsteilung gliedert die Aufgaben wie folgt:

Wissensmanager sind meist im Personalwesen verortet und erfüllen die Rolle des Projektmanagers bei der Einführung von Wissensvernetzung. Sie sind für die vorbereitenden Gespräche mit dem Senior Management verantwortlich und kümmern sich um die
Institutionalisierung der Communities. Wissensmanager übernehmen außerdem die Moderatorenrolle für die Community der CoP-Moderatoren und sind für das Coaching und die Koordinierung der Aktivitäten der Communities verantwortlich. Dabei sollte besonders auf die Erzeugung von Synergieeffekten zwischen den Communities geachtet werden. Wissensmanager leiten als Projekt- bzw. Arbeitsbereichsmanager Feedback und Anfragen der Teilnehmer bezüglich der NIKT-Werkzeuge an den Informationsmanager weiter und planen mit diesem die Weiterentwicklung der Systeme.

Informationsmanager sind am sinnvollsten innerhalb der IT-Abteilung beschäftigt. Sie sind Entwickler und Administratoren der Informations- und Kommunikationswerkzeuge und kümmern sich um die Anpassung der Funktionalität nach dem Feedback der Nutzer. Bei der Entwicklung des Kategorisierungssystems (Taxonomie) arbeiten die Informationsmanager eng mit den Wissensmanagern zusammen.

Um möglichst benutzerfreundliche und harmonische Arbeitsprozessabläufe für die Kommunikation und Kollaboration und Speicherung im Bereich Wissensvernetzung zu erreichen, sollten die Wissens- und Informationsmanager sehr eng zusammenarbeiten. Innerhalb eines Pilotprojekts können erste Erfahrungen, im Sinne von „Best Practice“, gesammelt werden. Auf dieser Grundlage lassen sich allgemeine Abläufe definieren, die aber für den Einsatz in unterschiedlichen Arbeitsbereichen flexibel auf die jeweiligen Arbeitsbedingungen der Teilnehmer angepasst werden müssen. Wichtig ist hierbei vor allem, dass jeder einzelne Arbeitsablauf genau dokumentiert und fuir alle Mitarbeiter zugänglich und vorzugsweise im Intranet zum Nachschlagen bereitgestellt wird.

Die Erstellung des Trainingsmaterials und die Durchfuihrung der Schulung im Bereich CSCW sollte von professionellen externen Fachkräften und/oder in Zusammenarbeit von Wissens- und Informationsmanagern umgesetzt werden.

\section{Anmerkungen/Literatur}

[1] Rory Chase; Creating a Knowledge Business Strategy, Management Trends International; 1998

[2] Claus Hoffmann: Das Intranet: Ein Medium der Mitarbeiterkommunikation. UVK Medien Verlagsgesellschaft, 2001, S. 107

[3] Institut für Arbeit und Technik im Wissenschaftszentrum Nordrhein-Westfalen, Strategische Wissensnetze: Wie Unternehmen die Ressource Wissen nutzen, Schneider 1999

[4] Manfred Perlitz: Internationales Management, 4. Auflage, UTB 2000, S 634

\section{Autor}

Dipl. Wirtschaftsinformatiker (FH) Maximilian Senges

Technische Fachhochschule Wildau

Tel. +496221866723

E-Mail: maxsenges@web.de 